\title{
Metformin against Cancer Stem Cells through the Modulation of Energy Metabolism: Special Considerations on Ovarian Cancer
}

\author{
Tae Hun Kim, ${ }^{1}$ Dong Hoon Suh, ${ }^{2}$ Mi-Kyung Kim, ${ }^{3}$ and Yong Sang Song ${ }^{4,5,6}$ \\ ${ }^{1}$ Department of Obstetrics and Gynecology, Korean Cancer Center Hospital, Korea Institute of Radiological and Medical Sciences, \\ Seoul 139-706, Republic of Korea \\ ${ }^{2}$ Department of Obstetrics and Gynecology, Seoul National University Bundang Hospital, Seongnam 463-707, Republic of Korea \\ ${ }^{3}$ Biomedical Science Project, Brain Korea 21 Program for Leading Universities \& Students, Seoul National University, \\ Seoul 110-799, Republic of Korea \\ ${ }^{4}$ Department of Obstetrics and Gynecology, Seoul National University College of Medicine, Seoul 110-744, Republic of Korea \\ ${ }^{5}$ Cancer Research Institute, Seoul National University College of Medicine, Seoul 110-799, Republic of Korea \\ ${ }^{6}$ Major in Biomodulation, World Class University, Seoul National University, Seoul 151-921, Republic of Korea
}

Correspondence should be addressed to Yong Sang Song; yssong@snu.ac.kr

Received 28 February 2014; Accepted 29 May 2014; Published 24 June 2014

Academic Editor: Daisuke Aoki

Copyright (C) 2014 Tae Hun Kim et al. This is an open access article distributed under the Creative Commons Attribution License, which permits unrestricted use, distribution, and reproduction in any medium, provided the original work is properly cited.

\begin{abstract}
Ovarian cancer is the most lethal gynecologic malignancy among women worldwide and is presumed to result from the presence of ovarian cancer stem cells. To overcome the limitation of current anticancer agents, another anticancer strategy is necessary to effectively target cancer stem cells in ovarian cancer. In many types of malignancies, including ovarian cancer, metformin, one of the most popular antidiabetic drugs, has been demonstrated to exhibit chemopreventive and anticancer efficacy with respect to incidence and overall survival rates. Thus, the metabolic reprogramming of cancer and cancer stem cells driven by genetic alterations during carcinogenesis and cancer progression could be therapeutically targeted. In this review, the potential efficacy and anticancer mechanisms of metformin against ovarian cancer stem cells will be discussed.
\end{abstract}

\section{Introduction}

Epithelial ovarian cancer (EOC) is the most lethal gynecologic malignancy and frequently presents with peritoneal carcinomatosis [1]. Approximately $60 \%$ to $70 \%$ of cases are diagnosed in advanced stages, and the five-year survival rate is $30 \%$ for advanced-stage ovarian cancer [2]. Although cytoreductive surgery and adjuvant chemotherapy are effective in extirpating tumor mass, approximately $80 \%$ of patients with advanced-stage EOC experience recurrence, after which chemotherapy is no longer as effective as it was previously [3]. Additionally, EOC exhibits high intratumoral heterogeneity at both the genotypic and phenotypic levels, with diverse biologic consequences [4]. The cancer stem cell (CSC) model is one of the emerging mechanisms of chemoresistance and intratumoral heterogeneity in EOC. CSCs, also called tumor initiating cells (TICs), are a subset of cancer cells within each tumor that exhibit the ability to induce tumors when transplanted into immune-deficient mice. CSCs are thought to constitute a small subset of cells within a tumor that initiate both the primary disease and its recurrence because of their capacity for self-renewal and inherent chemoresistance [5]. CSCs also possess properties of self-renewal as well as the ability to undergo serial passages in vitro and in vivo due to unlimited division potential [6].

Alterations in cellular bioenergetics are an emerging hallmark of cancer. The shift from oxidative phosphorylation to aerobic glycolysis (i.e., the Warburg effect) is the bestcharacterized metabolic phenotype of cancer [7]. Recent studies demonstrate that aerobic glycolysis is a characteristic of proliferating cells as well as the metabolic phenotype used by pluripotent cells, including CSCs [8]. Metformin is an oral antidiabetic drug in the biguanide class and the firstline drug of choice for the treatment of type 2 diabetes. Considering the association between CSCs and metabolic reprogramming, it is intriguing that metformin exhibits an 
anticancer effect, especially to CSCs [9-13]. However, the molecular mechanism by which metformin inhibits CSCs is unclear. This review will discuss the potential anticancer effect of metformin based on the hypotheses of CSC in the context of metabolic reprogramming in ovarian cancer.

\section{Cancer Stem Cells as a Cause of Chemoresistance in Ovarian Cancer}

The current first-line chemotherapy for EOC patients is a combination of carboplatin and paclitaxel [14]. Most relapses are likely the result of sparing of ovarian CSCs. Resistance to conventional chemotherapy has also been suggested to be a unique property of CSCs. Decreased responsiveness to chemotherapy might be due in part to the slow proliferation rates of CSCs, given that conventional cytotoxic drugs mainly target highly proliferative cells [15]. High expression of ATP-binding cassette drug transporters and antiapoptotic proteins, the ability to protect cells from DNA damage, and efficient DNA repair have been suggested to be a cause of chemoresistance of CSCs [16]. In addition, ovarian CSCs are resistant to tumor necrosis factor $\alpha$-mediated apoptosis [5].

Several studies have demonstrated that chemotherapytreated residual tumors are enriched in cells with a CSC phenotype, whereas cells lacking these characteristics are eliminated [17-20]. Steg et al. reported that a proportion of ovarian CSCs seem to be increased after chemotherapy [20]. Primary samples were composed of low densities of CSC markers, such as ALDH1A1, CD44, and CD133. Tumors collected immediately after primary therapy were more densely composed of each marker, whereas samples collected at the first recurrence, before initiating secondary therapy, were composed of similar percentages of each marker as the primary tumor. These results suggest that stem cell subpopulations contribute to tumor chemoresistance and ultimately recurrent disease [20].

The enrichment of CSCs after chemotherapy can be simply explained by the remaining chemoresistant CSC population. However, recent evidence suggests that chemotherapeutic treatment results in the generation of ovarian CSCs. Emerging evidence is suggesting that epithelialmesenchymal transition (EMT) plays a crucial role in chemoresistance and the generation of CSC populations [2124]. The EMT and CSC-like cell phenotypes are closely related responses to chemotherapy [25]. Kurrey et al. reported that EMT-induced expression of the E-cadherin transcriptional repressors Snail and Slug has been shown to impose the acquisition of the CSC-like phenotype and chemoresistance in EOC cells by defying p53-mediated apoptosis [21]. Lafti et al. reported that cisplatin treatment of primary and metastatic EOC generates residual cells with mesenchymal stem celllike profiles. The authors also demonstrated a significant enhancement in the sphere-forming abilities of ovarian cancer cells in response to chemotherapy drugs [19]. In addition, xenotransplantation studies using chemotherapytreated EOC cells generated significantly larger tumor burdens compared with untreated cells, had a greater proliferative and tumorigenic capacity, and retained an enhanced stemness profile as evidenced by the enhanced expression of Ki67, CA125, CD117, and Oct4 [19].

\section{Ovarian Cancer Stem Cell Model}

Many investigators have reported the identification and isolation of human ovarian CSCs based on either the differential expression of cell surface markers or differential biochemical properties. Accumulating evidence indicates that no single marker clearly identifies the ovarian CSC, and more recent reports have suggested that multiple cell populations defined by distinct marker profiles may in fact represent CSCs in EOC [26].

Since Bapat et al. first reported the isolation and identification of stem-like cells from the ascites of a patient with EOC [27], numerous putative cell surface and intracellular markers have been used to isolate and characterize ovarian CSCs. These include CD44, epithelial cell adhesion molecule (EpCAM), CD133, CD117, CD90 (Thy-1), CD24 [5, 28-31], and intracellular marker aldehyde dehydrogenase (ALDH) [32]. Ovarian CSCs can be successfully isolated via distinctive efflux of the DNA-binding dye Hoechst 33342. These ovarian CSCs are also called "side population" (SP) stem cells and have the capacity of self-renewal and differentiation in comparison with the non-SPs [33]. To date, the most commonly used markers for ovarian CSCs are CD133 and ALDH. ALDH $(+)$ cells are inherently resistant to chemotherapy [34]. Small numbers of ALDH (+) cells can initiate tumors in mice, whereas a 10 - to 50 -fold excess of ALDH (-) cells cannot. Interestingly, cells that express both ALDH and CD133 possess greater tumor initiation capacity [34]. The identification of ovarian CSCs with various markers has been comprehensively reviewed $[35,36]$.

3.1. CSC Phenotypes and Metabolic Reprogramming. Although the metabolic requirements of CSCs are not fully understood, recent evidence suggests that pluripotent stem cells (PSCs) and cancer cell metabolism are overtly similar.

3.1.1. Advantages of Aerobic Glycolysis: Pluripotency and Stemness. Briefly, rapidly proliferating non-CSC tumor cells are metabolically characterized by "aerobic glycolysis," that is, high glycolysis even in the presence of oxygen, which provides tumor cells with 3 advantages: macromolecular biosynthesis, tumor invasion, and chemoresistance [37]. Recently, our group demonstrated that the overexpression of hexokinase II was an independent risk factor for chemoresistance using 111 EOC specimens [38]. In this study, hexokinase II overexpression was also found to be associated with short progression-free survival.

On the other hand, the glycolytic phenotype of CSCs is likely to be associated with pluripotency and stemness. Numerous studies have demonstrated that mouse and human embryonal stem cells (ESCs) and induced PSCs (iPSCs) exhibit elevated dependence on glycolysis under aerobic conditions compared with differentiated cells, such as cardiomyocytes and fibroblasts [39-42]. Somatic cells reprogrammed to pluripotency should become dependent on glycolysis 
[43]. During the reprogramming process, an increase in the expression of specific glycolytic genes precedes the gain of expression in genes that regulate self-renewal, suggesting that metabolic resetting has an early, active role in the return to pluripotency [44]. In addition, low oxygen content also helps to maintain ESC self-renewal and increases iPSC reprogramming efficiency. The parallel metabolic changes in oncogenesis and the induction of pluripotency elicit the hypothesis that cell bioenergetics can operate as the pivotal decisionmaking parameter during the reprogramming acquisition of stem cell properties in normal and non-CSC tumor cells [8]. In addition to the rapid energy production and the generation of building blocks, the glycolytic phenotype has a more fundamental role in inducing stemness. Therefore, it was suggested that the Warburg effect is the permitted cell metabotype possessing the necessary plasticity to reprogram the tumor cell of origin so that it can acquire the cellular state of a CSC [8].

The glycolytic phenotype appears to be closely associated with stemness. Liao et al. reported that spheroid cells, which were enriched for cells with cancer stem cell-like characteristics, routed glucose predominantly to anaerobic glycolysis and the pentose cycle to the detriment of rerouting glucose for anabolic purposes [45]. However, there is controversy over whether CSCs exhibit a glycolytic phenotype. The group of researchers supporting the "reverse Warburg effect" suggested that CSCs may rely on oxidative phosphorylation (OXPHOS) based on the selective toxicity of metformin on CSCs [46].

3.2. CSC and Tumor Microenvironment. The CSC model provides one explanation for the phenotypic and functional heterogeneity among cancer cells in EOC. The reversible CSC phenotype continuously evolves and can be switched on or off in response to cell-intrinsic or microenvironmental cues, including therapeutics and hypoxia [8, 47]. Additionally, molecular cues from stromal cells provide the signaling to maintain and expand the stem cell phenotype. An analysis of epithelial and mesenchymal markers in EOC reveals phenotypic heterogeneity and plasticity, and this phenotypic plasticity was dependent on external factors, such as stress created by starvation or contact with either epithelial or mesenchymal cells in cocultures [48]. Abelson et al. reported that putative ovarian CSCs with different levels of morphologic and tumorigenic differentiation display microenvironment-dependent plasticity with the capacity to restore self-renewal and CD44 expression [49]. In addition to the fact that ovarian CSCs can induce the formation of an inflammatory environment [50], stromal cells can also influence the maintenance of CSCs. A 4- to 8-fold increase in the percentage of putative ovarian CSCs has been observed in the presence of carcinoma-associated mesenchymal stem cells both in vitro and in vivo [51].

Conversely, CSCs can influence the tumor microenvironment. In xenotransplantation studies, which are the gold standard studies for CSC models, a relatively small number of transplanted CSCs must encounter a hostile microenvironment with hypoxia conditions and a lack of nutrients [52].
To promote tumor development, CSCs must have the ability to modulate the tumor microenvironment. Nevertheless, CSCs are supposed to have the ability to proliferate without preestablished microenvironmental support such as growth signals, inflammatory factors, or nutrients. Autonomous inflammatory activation and a preference for hypoxia should be the required abilities of CSCs.

3.2.1. Inflammation and CSC. Ovarian CSCs induce inflammation in the microenvironment [50]. The chemoresistant subpopulation of EOC, through constitutive cytokine production, may significantly contribute to the maintenance of the inflammatory environment that promotes tissue repair and renewal [53]. The main trigger of constitutive nuclear factor kappa-light-chain-enhancer of activated B cells (NF$\kappa \mathrm{B}) /$ cytokine production is IKK $\beta$, which is expressed only in the chemoresistant subpopulation [54]. Ovarian CSCs isolated from ascites and solid tumors are characterized by the following: CD44+, MyD88+, constitutive NF- $\kappa$ B activity, cytokine and chemokine production, high capacity for repair, chemoresistance to conventional chemotherapies, resistance to TNF- $\alpha$-mediated apoptosis, capacity to form spheroids in suspension, and ability to recapitulate in vivo the original tumor [5]. Constitutive NF- $\kappa \mathrm{B}$ activity and the secretion of proinflammatory cytokines have been demonstrated in the CD44+ population but not in CD44- EOC cells [5]. On the other hand, autocrine secretion of transforming growth factor- (TGF- $\beta$ ) by EOC cells has been shown to be responsible for an EMT-mediated increase of the CD44+/CD117+ population [55]. This effect was enhanced when EOC cells were cultured on fibronectin, demonstrating once again the additive effect of the different components of the tumor microenvironment on CSCs [56].

3.2.2. Hypoxia and CSC. Hypoxia might be one of the key attributes in the tumor microenvironment that can regulate the phenotype of CSCs. Hypoxia was shown to maintain or upregulate ovarian CSC characteristics [57]. During hypoxia, anaerobic conditions prevail within the tumor, activating oncogenes such as MYC and RAS, resulting in the expression of hypoxia inducible factor- (HIF-) 1 and HIF-2 and the induction of the expression of pluripotent genes, such as Oct4, Sox3, and kruppel-like factor- (KLF-) 4 [47, 57]. In another study, hypoxia facilitated the survival of CD117-enriched CSCs in EOC cell lines and cells derived from primary ovarian tumors through the activation of Wnt/beta-catenin and the ATP-binding cassette G2 downstream of CD117 [58]. Hypoxia has also been shown to induce EMT differentiation in CD44+ My88+ enriched ovarian CSC-derived xenografts obtained from primary ovarian tumors and ascites [59].

3.3. Plasticity of CSC. In contrast to the traditional view of a one-way CSC hierarchy in which CSCs give rise to nonCSCs but not vice versa, resulting in a hierarchical celllineage structure reflective of normal tissue biology $[30,60]$, the phenotypic plasticity of CSC was recently demonstrated. Gupta et al. recently reported that subpopulations of cells purified for a given phenotypic state from a breast cancer cell 


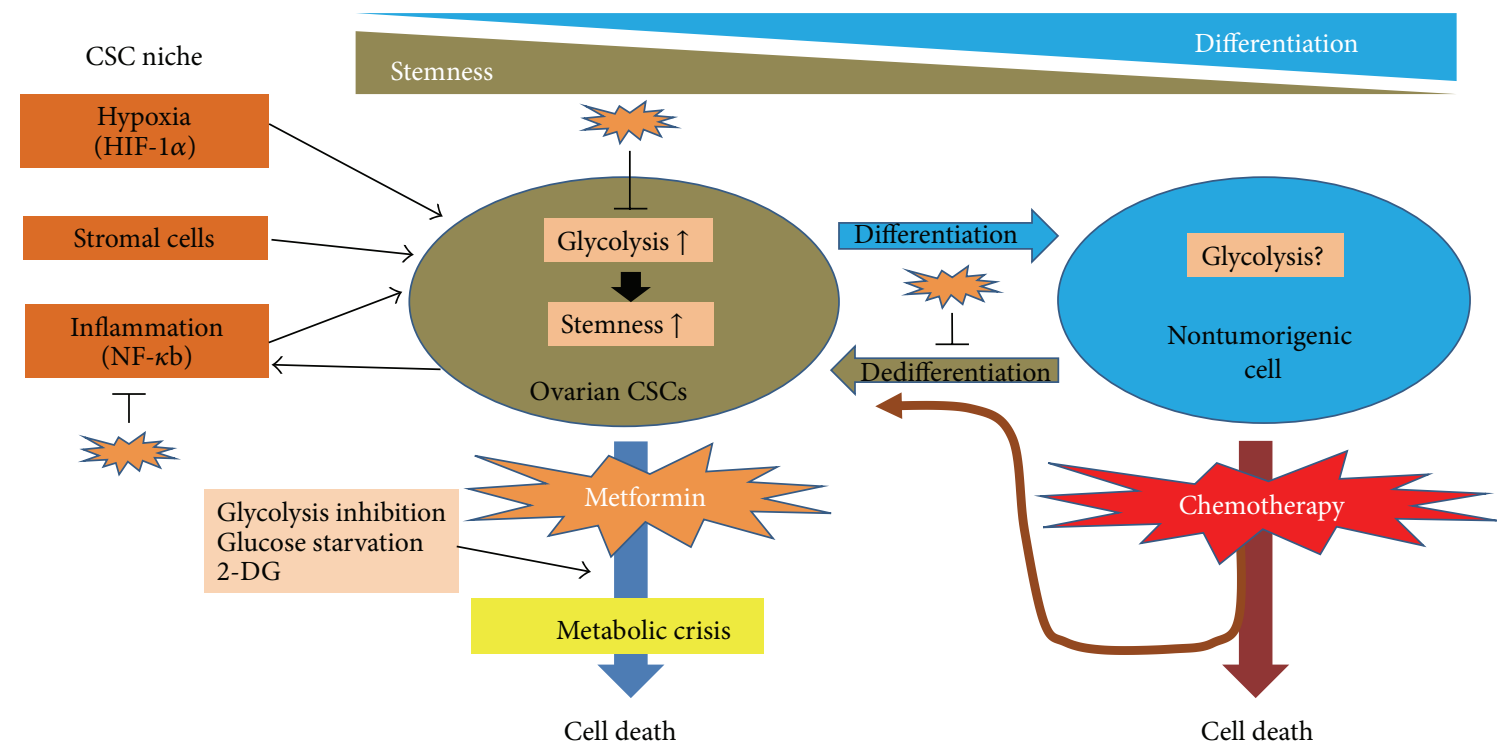

inns Metformin

FIGURE 1: Mechanism of action of metformin in the concept of dynamic cancer stem cells in ovarian cancer. In contrast to the hierarchically organized cancer stem cell (CSC) model, the dynamic CSC model represents bidirectional interconversion between CSC and differentiated non-CSC states. The acquisition and maintenance of CSC characteristics are affected by microenvironmental cues, including inflammation, stromal cells and hypoxia, and therapeutics, such as chemotherapy. These factors eventually constitute the CSC niche (gray field). Hypoxia, which causes glycolysis, maintains and upregulates ovarian CSCs characteristics. Chemotherapy kills rapidly proliferating nontumorigenic cells, sparing chemoresistant CSCs. Chemotherapy also induces the acquisition of stem cell characteristics via epithelial-mesenchymal transition. Many studies using embryonal stem cells and induced pluripotent stem cells have demonstrated that glycolysis plays a fundamental role in inducing stemness. It is hypothesized that glycolysis may have a critical role in acquiring the CSC phenotype. It remains to be elucidated whether the metabotype is different between tumorigenic CSCs and rapidly proliferating nontumorigenic cells. Assumptive mechanisms of metformin's synergic effect on chemotherapy and selective toxicity to CSCs are illustrated. Metabolic stress caused by metformin may inhibit the transition to the glycolytic phenotype, resulting in the prevention of the acquisition of stemness and dedifferentiation. Metformin may also target the inflammatory components present in the tumor microenvironment. Ovarian CSCs may lack the ability to cope with metabolic stress caused by metformin and glucose starvation.

line return towards equilibrium proportions over time [61]. The authors proposed an alternative scenario in which there is bidirectional interconversion between CSC and non-CSC states [61].

Although many researchers have reported the clinical implication of targeting CSC surface markers, the fact that the ovarian CSC phenotype was not a consistent state but a changeable state depending on external conditions has been overlooked [36]. An emerging consensus in the field is that the cellular state rather than the cellular phenotype is crucial to defining and investigating CSCs [8]. However, the phenotypic plasticity of CSC and its dependence on the microenvironment do not imply that CSCs are illusive targets in cancer therapy but convince us that we must focus on where CSCs originate and how CSCs are generated and maintained under various conditions.

\section{Metformin: A Promising Metabolic Approach Targeting CSCs in Ovarian Cancer}

Many in vitroand in vivostudies have demonstrated the antiproliferative action of metformin on various cancer cell lines and animal cancer models (Table 1 and Figure 1) $[13,62-$ 65]. This review will focus on metformin in detail.

4.1. Metformin on Cancer Stem Cells. The selective toxicity of metformin on CSC has been reported in various cancers. Selective inhibition of CSCs by metformin was first reported in 2009 in preclinical breast cancer models [11]. These results were subsequently extended to cancer cell lines from prostate and lung adenocarcinomas, where metformin similarly inhibited CSCs [68]. Recently, a selective toxicity on CSCs was also reported in EOC [13]. Metformin was shown to act on ovarian CSCs, reducing the percentage of ALDH (+) CSC in vitro and in vivo and inhibiting the growth of ovarian tumor spheres. Metformin was active against primary human ovarian CSCs in vitro, and metformin therapy alone slowed the growth of ovarian CSC in vivo [13].

Although the molecular mechanism by which metformin inhibits the self-renewal of CSCs is still obscure, it is noteworthy that iPSCs downregulate the expression of the catalytic subunit of the AMP-activated protein kinase (AMPK), which is a negative regulator for anabolic processes [69]. Activation of AMPK provides a metabolic barrier to reprogramming 
TABLE 1: Preclinical studies of metformin targeting metabolism of ovarian cancer cells and/or ovarian CSCs.

\begin{tabular}{|c|c|c|c|c|c|}
\hline Agent or drug (dosage) & $\begin{array}{c}\text { CSCs } \\
\text { enriched }\end{array}$ & Alone & $\begin{array}{c}\text { Combination with other } \\
\text { drugs }\end{array}$ & Mechanism of action & Reference \\
\hline $\begin{array}{l}\text { Metformin } \\
(0.02 \mathrm{mmol} / \mathrm{L} \text { in vitro })\end{array}$ & No & $\begin{array}{l}\text { No significant loss of } \\
\text { viability or change in cell } \\
\text { cycle }\end{array}$ & $\begin{array}{l}\text { Improvement of cytotoxic } \\
\text { response to carboplatin }\end{array}$ & $<->$ & {$[63]$} \\
\hline $\begin{array}{l}\text { Metformin } \\
\text { ( } 5 \mathrm{mmol} / \mathrm{L} \text { in vitro), } \\
\text { PEITC } \\
(5 \mu \mathrm{mol} / \mathrm{L} \text { in vitro })\end{array}$ & No & $\begin{array}{l}\text { Inhibition of growth in } \\
\text { vitro }\end{array}$ & $\begin{array}{l}\text { Combination with PEITC } \\
\text { increases cell death in vitro }\end{array}$ & ROS generation & {$[62]$} \\
\hline $\begin{array}{l}\text { Metformin } \\
(5 \mathrm{mmol} / \mathrm{L} \text { in vitro })\end{array}$ & No & Induced apoptosis in vitro & $\begin{array}{l}\text { Combination with cisplatin } \\
\text { enhances apoptosis }\end{array}$ & $\begin{array}{c}\text { AMPK-independent, } \\
\text { downregulating } \\
\text { Bcl-2/Bcl-xL, upregulating } \\
\text { Bax/Bad }\end{array}$ & {$[65]$} \\
\hline $\begin{array}{l}\text { Metformin } \\
(100-200 \mathrm{mg} / \mathrm{kg} \text { in vivo })\end{array}$ & No & $\begin{array}{l}\text { Inhibition of ovarian tumor } \\
\text { growth, proliferation, } \\
\text { metastasis, and } \\
\text { angiogenesis in vivo }\end{array}$ & $\begin{array}{l}\text { Combination with cisplatin } \\
\text { reduces tumor growth }\end{array}$ & $\begin{array}{c}\text { AMPK/mTOR, } \\
\text { antiangiogenic effect }\end{array}$ & {$[64]$} \\
\hline $\begin{array}{l}\text { Metformin } \\
(5 \mathrm{mmol} / \mathrm{L} \text { in vitro })\end{array}$ & No & $\begin{array}{l}\text { Inhibition of proliferation } \\
\text { in vitro }\end{array}$ & $<->$ & $\begin{array}{c}\text { Cell cycle arrest, } \\
\text { AMPK/mTOR and AMPK } \\
\text { independent pathway }\end{array}$ & {$[66]$} \\
\hline $\begin{array}{l}\text { Metformin } \\
(5-50 \mathrm{mmol} / \mathrm{L} \text { in vitro })\end{array}$ & No & $\begin{array}{l}\text { Inhibition of proliferation } \\
\text { in vitro }\end{array}$ & $\begin{array}{l}\text { Improvement of cytotoxic } \\
\text { response to cisplatin }\end{array}$ & AMPK/mTOR & {$[67]$} \\
\hline $\begin{array}{l}\text { Metformin } \\
(0.3 \mathrm{mmol} / \mathrm{L} \text { in vitro, } \\
150 \mathrm{mg} / \mathrm{kg} \text { in vivo })\end{array}$ & $\begin{array}{l}\text { ALDH+ } \\
\text { cells }\end{array}$ & $\begin{array}{c}\text { Inhibition of ovarian } \\
\text { CSC/TIC growth in vitro, } \\
\text { nonsignificant decreases in } \\
\text { tumor growth in vivo }\end{array}$ & $\begin{array}{l}\text { Combination with cisplatin } \\
\text { restricts tumor growth in } \\
\text { vivo }\end{array}$ & $<->$ & {$[13]$} \\
\hline
\end{tabular}

CSC, cancer stem cell; ROS, reactive oxygen species; AMPK, adenosine monophosphate-activated protein kinase; mTOR, mammalian target of rapamycin; ALDH, aldehyde dehydrogenase; PEITC, phenethyl isothiocyanate; TIC, tumor initiating cell. Modified and adapted with permission from reference [15].

somatic cells into stem cells [70]. The AMPK activators established a metabolic barrier to reprogramming that could not be bypassed, even through 533 deficiency, a fundamental mechanism to greatly improve the efficiency of stem cell production. Monitoring the transcriptional activation status of each individual reprogramming factor (i.e., Oct4, Sox2, $\mathrm{Klf4}$, and c-Myc) revealed that AMPK activation could prevent the transcriptional activation of Oct4, the master regulator of the pluripotent state. AMPK activation appears to impose a normalized metabolic flow away from the required proimmortalizing glycolysis that fuels the induction of stemness and pluripotency, endowing somatic cells with an energetic infrastructure that is protected against reprogramming. Decreased AMPK expression correlated significantly with higher tumor grade and was of adverse prognosis in EOC [71].

4.2. Clinical Evidence of Metformin in the Prevention and Treatment of EOC. A meta-analysis concluded that patients with diabetes exhibited a statistically significant increased risk of EOC [72]. Type 2 diabetic patients with EOC have poor survival outcomes and are more likely to exhibit poorly differentiated tumor histology compared with nondiabetic EOC patients [73]. These findings can be simply explained by the growth-promoting effect of chronic elevated plasma insulin levels and persistent elevated plasma glucose levels [74]. On the other hand, metformin has been demonstrated to have a chemopreventive and anticancer effect in many types of malignancies, including $\operatorname{EOC~}[75,76]$. In a recent meta-analysis, metformin exhibited a tendency to reduce the occurrence of EOC among diabetic patients with the pooled odds ratio of 0.57 (95\% confidence interval, 0.16-1.99) [77]. Furthermore, Kumar et al. reported in a case-control study that metformin intake was associated with a better survival in EOC [76]. Interestingly, metformin-associated tumors were more likely to be of lower grade and earlier stage and had a more favorable histology profile [76].

\subsection{Mechanism of Action of Metformin in the Prevention and Treatment of EOC}

4.3.1. Insulin and IGF-1. It has not been clearly confirmed how metformin decreases cancer incidence and prolongs the survival of cancer patients. A number of possible mechanisms regarding the anticancer effects of metformin have been suggested (Figure 2). The association between metformin and a reduced risk of cancer in diabetic patients may simply be explained through the action of metformin on the improvement in blood glucose and insulin levels because hyperinsulinemia and hyperglycemia play an important role in cancer proliferation $[78,79]$. By decreasing the circulating levels of insulin and IGF-1, metformin may ameliorate this negative effect of hyperinsulinemia in diabetic patients. 


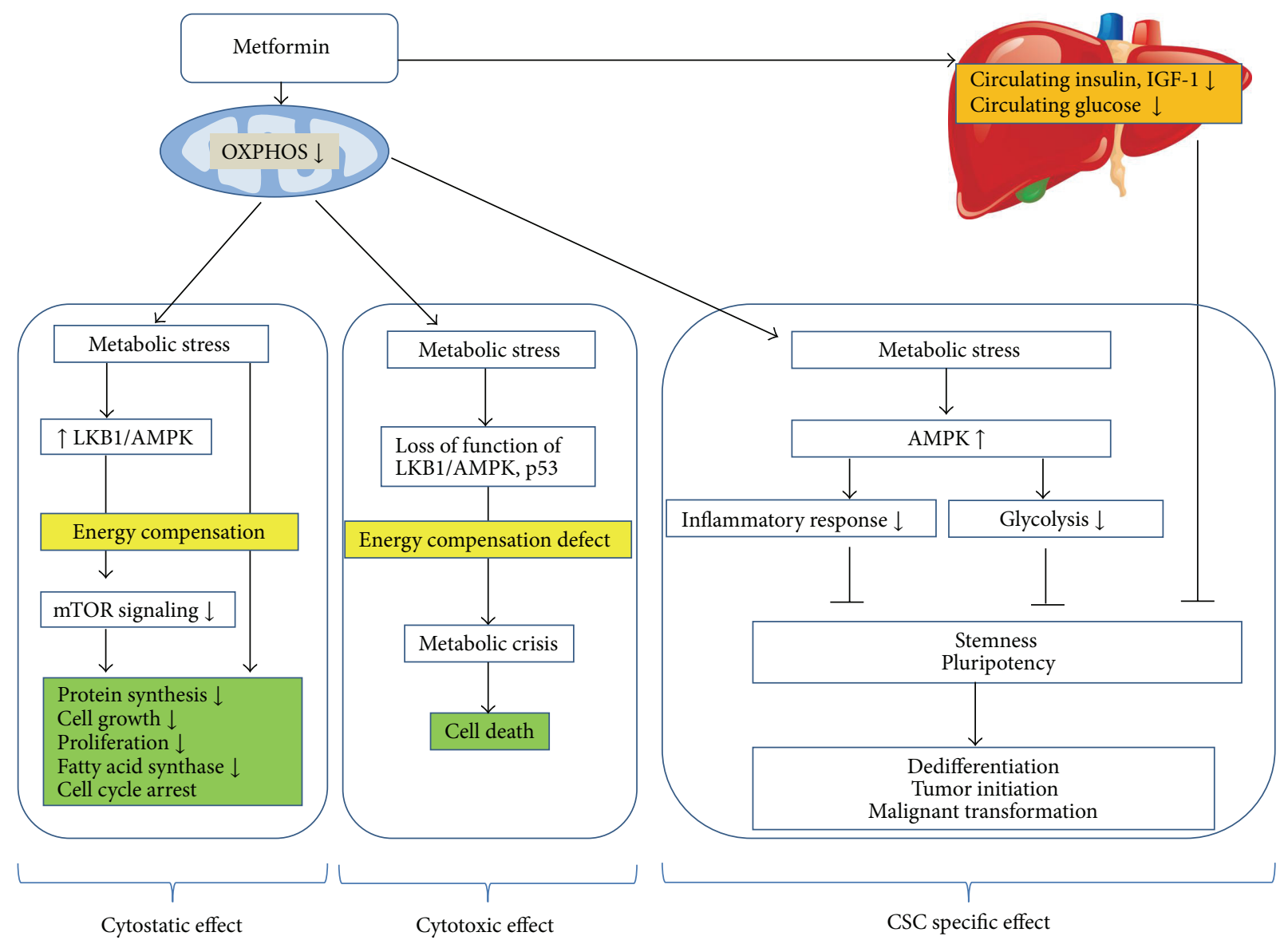

FIGURE 2: Antineoplastic mechanisms of action of metformin. The mitochondria are the primary target of metformin. Metformin interferes with oxidative phosphorylation via interactions with respiratory complex I, resulting in reduced ATP production and metabolic stress. Metformin lowers plasma glucose levels by decreasing gluconeogenesis and glucose uptake, resulting in lower circulating insulin and IGF1 levels. An energy compensation reaction occurring in tumor cells capable of responding to metabolic stress is shown in the left box. By activating the LKB1/AMPK pathway, metformin inhibits mTOR downstream signaling, ultimately causing downregulation of energyconsuming processes and an overall cytostatic effect. The antitumour effects of metformin are regulated by both AMPK-dependent and AMPK-independent mechanisms. If tumor cells lack ability to cope with energetic stress due to the loss of function of LKB1/AMPK or p53, they may undergo a metabolic crisis leading to death (middle box). The right box presents an assumptive mechanism of metformin's action against CSCs. Both inflammation and the glycolytic phenotype are likely to be associated with pluripotency and stemness. The activation of AMPK provides a metabolic barrier to reprogramming somatic cells into stem cells. Metformin has been demonstrated to inhibit dedifferentiation processes, tumor initiation, and malignant transformation. Anti-inflammatory effects, restoration from glycolysis, and reduced growth signals might explain the anti-CSC action of metformin.

4.3.2. Metformin Induces Metabolic Stress. Metformin's primary activity is the inhibition of complex I of the mitochondrial electron-transport chain, resulting in an increase of the intracellular AMP/ADP ratio and thereby activating AMPK, a negative regulator of anabolic process (Figure 2) [80]. The inhibition of OXPHOS leads to lower ATP levels and reprogramming of cellular energy metabolism in favor of conserving energy and restoring ATP levels, ultimately causing downregulation of energy-consuming processes and an overall cytostatic effect [81]. Metformin appears to have a direct action on tumor growth both in vitroand in vivoby a mechanism involving the activation of the LKB1/AMPK pathway and the subsequent modulation of downstream pathways controlling cellular proliferation. The antineoplastic activity of metformin via AMPK activation is mediated through the inhibition of mTORC1 signaling, leading to inhibition of protein synthesis and cell proliferation [81]. mTOR plays a major role in carcinogenesis, and its activation is linked with cancer progression and poor outcomes in EOC [82].

Metformin induces metabolic stress by reducing mitochondrial ATP production. Therefore, it has been suggested that metformin could inhibit the growth of cancer cells by decreasing the cellular energy status and force a metabolic conversion that cancer cells are unable to execute [83]. A study revealed that AMPK activation promotes the survival of cells metabolically impaired by glucose limitation in part through p53 activation [84]. Moreover, loss of p53 impairs the ability of cancer cells to respond to metabolic changes induced by metformin and to survive under conditions of 
nutrient deprivation [85]. Furthermore, LKB1-deficient cells were more sensitive to metformin-induced energy stress when cultured at low glucose concentrations and were unable to compensate for the decreased cellular ATP concentration, causing cell death [86]. These cytotoxic effects of metformin arise only in the context of a genetic defect, such as loss of p53 and/or LKB1, that is present in the cancer but not in the normal host tissue, providing opportunities for "synthetic lethality" [81]. Glucose deprivation induces metabolic stress, resulting in AMPK activation. 2-Deoxyglucose (2DG), a wellknown glycolysis inhibitor, can induce apoptosis in combination with metformin in various cancer cell lines, including EOC [87-89]. Cheong et al. reported that 2DG and metformin led to significant cell death associated with a decrease in cellular ATP, prolonged activation of AMPK, and sustained autophagy [88]. Importantly, forced energy restoration with methyl pyruvate reversed the cell death induced by 2DG and metformin, suggesting that prolonged activation of AMPK by $2 \mathrm{DG}$ and metformin might reflect sustained bioenergetic stress due to the failure of mitochondrial compensation.

Recent studies have revealed the existence of an alternative AMPK-independent pathway. Rattan et al. demonstrated that metformin treatment can arrest the cell cycle, decrease cyclin D1 expression, increase p21 protein expression, attenuate mTOR-S6 RP phosphorylation, and inhibit protein-translational and lipid biosynthetic pathways in EOC [66]. Although these antineoplastic effects of metformin coexisted with the activation of the LKB1/AMPK pathway, the effects were reproduced in AMPK-silenced EOC cells but not in LKB1 inactivated cells [66]. Therefore, it was suggested that LKB1 may have pivotal role in metformin's antineoplastic action. In fact, loss of the LKB1 and PTEN tumor suppressor genes in the ovarian surface epithelium was reported to induce papillary serous ovarian cancer [90]. Metformin induced apoptosis in EOC cell lines in an AMPKindependent manner and provoked a cell cycle arrest in the $\mathrm{S}$ and $\mathrm{G} 2 / \mathrm{M}$ phase [65].

4.3.3. Inflammation. Another potential mechanism is based on the positive impact of metformin on chronic inflammation. The role of chronic inflammation in promoting ovarian tumorigenesis and cancer progression has been well demonstrated elsewhere [91]. Metformin has been shown to decrease the production of inflammatory cytokines, including TNF- $\alpha$, interleukin-6, and vascular endothelial growth factor, through the inactivation of NF- $\kappa \mathrm{B}$ and HIF- $1 \alpha$ [9294]. Emerging results demonstrating the capacity of AMPK to inhibit the inflammatory responses suggest that metformin may also target the inflammatory component present in the tumor microenvironment [95]. In addition, several reports demonstrated that metformin treatment inhibits neoplastic angiogenesis, resulting in the reduction of tumor growth $[13,64,96,97]$. Wu et al. reported that metformin inhibits the development and metastasis of EOC by reducing neovascularization and macrophage infiltration [96].

4.3.4. Reactive Oxygen Species (ROS). Complex I inhibition is partially involved in metformin's growth inhibition of EOC, possibly by increasing ROS and sensitizing cancer to additional oxidative stress. Phenethyl isothiocyanate (PEITC) induces EOC cell death by increasing ROS. When given together, metformin and PEITC exhibit a synergistic increase in cell death in several EOC cell lines, including cisplatinresistant cell lines [62].

4.4. Synergistic Anticancer Effects of Metformin and Chemotherapeutic Agents. Metformin has been demonstrated to augment the effects of various chemotherapeutic regimens by improving their efficacy as well as overcoming the chemoresistance in EOC (Table 1) [63-65, 67]. In fact, most in vitro studies used doses of metformin between 1 and $40 \mathrm{mM}$, which is well above the feasible therapeutic plasma levels $(2.8-15 \mu \mathrm{M})$ in humans [98]. Whereas the cytotoxic effect of metformin alone was achieved at millimolar concentrations in most studies, Erices et al. observed cytotoxicity with micromolar metformin in combination with chemotherapy at concentrations where the chemotherapy alone produced no loss in viability [63]. The exact mechanism of the synergetic effect of metformin on chemotherapy has not been well illustrated in most studies. One of the explanations is that metformin might be selectively toxic to CSC, which has been regarded as the cause of chemoresistance. As mentioned above, metformin could selectively target CSCs and act together with chemotherapy to block tumor growth and prolong remission in breast cancer $[11,68]$, pancreatic cancer [99], and EOC [13].

\section{Conclusion}

CSCs are believed to be one of the main causes of chemoresistance because CSCs are quiescent but possess clonogenicity on their own. Recent studies have demonstrated a dominant role for the tumor microenvironment in determining CSC characteristics within a malignancy. Although the metabolic phenotype of CSCs is not well defined and differs significantly between types of cancer, metformin is thought to represent an emerging lethal weapon against CSCs in ovarian cancer on the basis of the capability to control the CSC niche as well as metabolic modulation. Nevertheless, there seems to be much to be elucidated regarding better characterization of CSCs as well as the interaction between CSC and the CSC niche moleculogenetically and metabolically. Further research of metformin is urgently required for effectively overcoming the chemoresistance of EOC by selectively targeting the metabolic features of ovarian CSCs.

\section{Conflict of Interests}

The authors declare that there is no conflict of interests regarding the publication of this paper.

\section{Acknowledgments}

This research was supported by a Grant from Doosan Yonkang Foundation (no. 30-2011-0070), Priority Research Centers Program (2009-0093820), Basic Science Research 
Program (2011-0025394), and WCU (World Class University) Program (R31-10056) through the National Research Foundation of Korea (NRF) funded by the Ministry of Education, Science and Technology.

\section{References}

[1] R. Siegel, D. Naishadham, and A. Jemal, "Cancer statistics, 2013," CA Cancer Journal for Clinicians, vol. 63, no. 1, pp. 11-30, 2013.

[2] N. Akhtar-Danesh, A. Lytwyn, and L. Elit, "Five-year trends in mortality indices among gynecological cancer patients in Canada," Gynecologic Oncology, vol. 127, no. 3, pp. 620-624, 2012.

[3] R. F. Ozols, B. N. Bundy, B. E. Greer et al., "Phase III trial of carboplatin and paclitaxel compared with cisplatin and paclitaxel in patients with optimally resected stage III ovarian cancer: a Gynecologic Oncology Group study," Journal of Clinical Oncology, vol. 21, no. 17, pp. 3194-3200, 2003.

[4] V. Wang, C. Li, M. Lin et al., "Ovarian cancer is a heterogeneous disease," Cancer Genetics and Cytogenetics, vol. 161, no. 2, pp. 170-173, 2005.

[5] A. B. Alvero, R. Chen, H.-H. Fu et al., "Molecular phenotyping of human ovarian cancer stem cells unravel the mechanisms for repair and chemo-resistance," Cell Cycle, vol. 8, no. 1, pp. 158166, 2009.

[6] M. R. Alison, W.-R. Lin, S. M. L. Lim, and L. J. Nicholson, "Cancer stem cells: in the line of fire," Cancer Treatment Reviews, vol. 38, no. 6, pp. 589-598, 2012.

[7] C. V. Dang, "Links between metabolism and cancer," Genes and Development, vol. 26, no. 9, pp. 877-890, 2012.

[8] J. A. Menendez, J. Joven, S. Cufí et al., "The warburg effect version 2.0: metabolic reprogramming of cancer stem cells," Cell Cycle, vol. 12, no. 8, pp. 1166-1179, 2013.

[9] R. Rattan, R. Ali Fehmi, and A. Munkarah, "Metformin: an emerging new therapeutic option for targeting cancer stem cells and metastasis," Journal of Oncology, vol. 2012, Article ID 928127, 12 pages, 2012.

[10] F. Bednar and D. M. Simeone, "Metformin and cancer stem cells: old drug, new targets," Cancer Prevention Research, vol. 5, no. 3, pp. 351-354, 2012.

[11] H. A. Hirsch, D. Iliopoulos, P. N. Tsichlis, and K. Struhl, "Metformin selectively targets cancer stem cells, and acts together with chemotherapy to block tumor growth and prolong remission," Cancer Research, vol. 69, pp. 7507-7511, 2009.

[12] H. A. Hirsch, D. Iliopoulos, and K. Struhl, "Metformin inhibits the inflammatory response associated with cellular transformation and cancer stem cell growth," Proceedings of the National Academy of Sciences of the United States of America, vol. 110, no. 3, pp. 972-977, 2013.

[13] J. J. Shank, K. Yang, J. Ghannam et al., "Metformin targets ovarian cancer stem cells in vitro and in vivo," Gynecologic Oncology, vol. 127, no. 2, pp. 390-397, 2012.

[14] S. Pignata, G. Scambia, G. Ferrandina et al., "Carboplatin plus paclitaxel versus carboplatin plus pegylated liposomal doxorubicin as first-line treatment for patients with ovarian cancer: the MITO-2 randomized phase III trial," Journal of Clinical Oncology, vol. 29, no. 27, pp. 3628-3635, 2011.

[15] M. J. Kwon and Y. K. Shin, "Regulation of ovarian cancer stem cells or tumor-initiating cells," International Journal of Molecular Sciences, vol. 14, no. 4, pp. 6624-6648, 2013.
[16] B.-B. S. Zhou, H. Zhang, M. Damelin, K. G. Geles, J. C. Grindley, and P. B. Dirks, "Tumour-initiating cells: challenges and opportunities for anticancer drug discovery," Nature Reviews Drug Discovery, vol. 8, no. 10, pp. 806-823, 2009.

[17] S. Rizzo, J. M. Hersey, P. Mellor et al., "Ovarian cancer stem celllike side populations are enriched following chemotherapy and overexpress EZH2," Molecular Cancer Therapeutics, vol. 10, no. 2, pp. 325-335, 2011.

[18] A. Latifi, R. B. Luwor, M. Bilandzic et al., "Isolation and characterization of tumor cells from the ascites of ovarian cancer patients: molecular phenotype of chemoresistant ovarian tumors," PLoS ONE, vol. 7, no. 10, Article ID e46858, 2012.

[19] K. Abubaker, A. Latifi, R. Luwor et al., "Short-term single treatment of chemotherapy results in the enrichment of ovarian cancer stem cell-like cells leading to an increased tumor burden," Molecular Cancer, vol. 12, no. 1, article 24, 2013.

[20] A. D. Steg, K. S. Bevis, A. A. Katre et al., "Stem cell pathways contribute to clinical chemoresistance in ovarian cancer," Clinical Cancer Research, vol. 18, no. 3, pp. 869-881, 2012.

[21] N. K. Kurrey, S. P. Jalgaonkar, A. V. Joglekar et al., "Snail and slug mediate radioresistance and chemoresistance by antagonizing p53-mediated apoptosis and acquiring a stem-like phenotype in ovarian cancer cells," Stem Cells, vol. 27, no. 9, pp. 2059-2068, 2009.

[22] N. Ahmed, K. Abubaker, J. Findlay, and M. Quinn, "Epithelial mesenchymal transition and cancer stem cell-like phenotypes facilitate chemoresistance in recurrent ovarian cancer," Current Cancer Drug Targets, vol. 10, no. 3, pp. 268-278, 2010.

[23] A. Latifi, K. Abubaker, N. Castrechini et al., "Cisplatin treatment of primary and metastatic epithelial ovarian carcinomas generates residual cells with mesenchymal stem cell-like profile," Journal of Cellular Biochemistry, vol. 112, no. 10, pp. 2850-2864, 2011.

[24] R. Y.-J. Huang, V. Y. Chung, and J. P. Thiery, “Targeting pathways contributing to epithelial-mesenchymal transition (EMT) in epithelial ovarian cancer," Current Drug Targets, vol. 13, no. 13, pp. 1649-1653, 2012.

[25] N. Ahmed, K. Abubaker, J. Findlay, and M. Quinn, "Cancerous ovarian stem cells: obscure targets for therapy but relevant to chemoresistance," Journal of Cellular Biochemistry, vol. 114, no. 1, pp. 21-34, 2013.

[26] R. Foster, R. J. Buckanovich, and B. R. Rueda, "Ovarian cancer stem cells: working towards the root of stemness," Cancer Letters, vol. 338, no. 1, pp. 147-157, 2013.

[27] S. A. Bapat, A. M. Mali, C. B. Koppikar, and N. K. Kurrey, "Stem and progenitor-like cells contribute to the aggressive behavior of human epithelial ovarian cancer," Cancer Research, vol. 65, no. 8, pp. 3025-3029, 2005.

[28] G. Ferrandina, E. Martinelli, M. Petrillo et al., "CD133 antigen expression in ovarian cancer," BMC Cancer, vol. 9, article 221, 2009.

[29] G. Ferrandina, G. Bonanno, L. Pierelli et al., "Expression of CD133-1 and CD133-2 in ovarian cancer," International Journal of Gynecological Cancer, vol. 18, no. 3, pp. 506-514, 2008.

[30] M.-Q. Gao, Y.-P. Choi, S. Kang, J. H. Youn, and N.-H. Cho, "CD24+ cells from hierarchically organized ovarian cancer are enriched in cancer stem cells," Oncogene, vol. 29, no. 18, pp. 2672-2680, 2010.

[31] S. Zhang, C. Balch, M. W. Chan et al., "Identification and characterization of ovarian cancer-initiating cells from primary human tumors," Cancer Research, vol. 68, no. 11, pp. 4311-4320, 2008 . 
[32] C. N. Landen Jr., B. Goodman, A. A. Katre et al., "Targeting aldehyde dehydrogenase cancer stem cells in ovarian cancer," Molecular Cancer Therapeutics, vol. 9, no. 12, pp. 3186-3199, 2010.

[33] M. A. Goodell, S. McKinney-Freeman, and F. D. Camargo, "Isolation and characterization of side population cells," Methods in Molecular Biology, vol. 290, pp. 343-352, 2005.

[34] I. A. Silva, S. Bai, K. McLean et al., "Aldehyde dehydrogenase in combination with CD133 defines angiogenic ovarian cancer stem cells that portend poor patient survival," Cancer Research, vol. 71, no. 11, pp. 3991-4001, 2011.

[35] D. Burgos-Ojeda, B. R. Rueda, and R. J. Buckanovich, “Ovarian cancer stem cell markers: prognostic and therapeutic implications," Cancer Letters, vol. 322, no. 1, pp. 1-7, 2012.

[36] Q. Zhan, C. Wang, and S. Ngai, "Ovarian cancer stem cells: a new target for cancer therapy," BioMed Research International, vol. 2013, Article ID 916819, 10 pages, 2013.

[37] O. Warburg, "On the origin of cancer cells," Science, vol. 123, no. 3191, pp. 309-314, 1956.

[38] D. H. Suh, M. A. Kim, H. Kim et al., "Association of overexpression of hexokinase II with chemoresistance in epithelial ovarian cancer," Clinical and Experimental Medicine, 2013.

[39] S. Chung, P. P. Dzeja, R. S. Faustino, C. Perez-Terzic, A. Behfar, and A. Terzic, "Mitochondrial oxidative metabolism is required for the cardiac differentiation of stem cells," Nature Clinical Practice Cardiovascular Medicine, vol. 4, 1, pp. S60-S67, 2007.

[40] C. D. L. Folmes, T. J. Nelson, A. Martinez-Fernandez et al., "Somatic oxidative bioenergetics transitions into pluripotencydependent glycolysis to facilitate nuclear reprogramming," Cell Metabolism, vol. 14, no. 2, pp. 264-271, 2011.

[41] A. D. Panopoulos, O. Yanes, S. Ruiz et al., "The metabolome of induced pluripotent stem cells reveals metabolic changes occurring in somatic cell reprogramming," Cell Research, vol. 22, no. 1, pp. 168-177, 2012.

[42] J. Zhang, I. Khvorostov, J. S. Hong et al., "UCP2 regulates energy metabolism and differentiation potential of human pluripotent stem cells," EMBO Journal, vol. 30, no. 24, pp. 4860-4873, 2011.

[43] J. Zhang, E. Nuebel, G. Q. Daley, C. M. Koehler, and M. A. Teitell, "Metabolic regulation in pluripotent stem cells during reprogramming and self-renewal," Cell Stem Cell, vol. 11, no. 5, pp. 589-595, 2012.

[44] C. D. Folmes, T. J. Nelson, and A. Terzic, "Energy metabolism in nuclear reprogramming," Biomarkers in Medicine, vol. 5, no. 6, pp. 715-729, 2011.

[45] J. Liao, F. Qian, N. Tchabo et al., "Ovarian cancer spheroid cells with stem cell-like properties contribute to tumor generation, metastasis and chemotherapy resistance through hypoxiaresistant metabolism," PLoS ONE, vol. 9, Article ID e84941, 2014.

[46] U. E. Martinez-Outschoorn, M. Prisco, A. Ertel et al., "Ketones and lactate increase cancer cell "stemness", driving recurrence, metastasis and poor clinical outcome in breast cancer: achieving personalized medicine via metabolo-genomics," Cell Cycle, vol. 10, no. 8, pp. 1271-1286, 2011.

[47] J. M. Heddleston, Z. Li, J. D. Lathia, S. Bao, A. B. Hjelmeland, and J. N. Rich, "Hypoxia inducible factors in cancer stem cells," British Journal of Cancer, vol. 102, no. 5, pp. 789-795, 2010.

[48] R. Strauss, Z.-Y. Li, Y. Liu et al., "Analysis of epithelial and mesenchymal markers in ovarian cancer reveals phenotypic heterogeneity and plasticity," PLoS ONE, vol. 6, no. 1, Article ID e16186, 2011.
[49] S. Abelson, Y. Shamai, L. Berger, R. Shouval, K. Skorecki, and M. Tzukerman, "Intratumoral heterogeneity in the self-renewal and tumorigenic differentiation of ovarian cancer," Stem Cells, vol. 30, no. 3, pp. 415-424, 2012.

[50] G. Mor, G. Yin, I. Chefetz, Y. Yang, and A. Alvero, "Ovarian cancer stem cells and inflammation," Cancer Biology and Therapy, vol. 11, no. 8, pp. 708-713, 2011.

[51] K. McLean, Y. Gong, Y. Choi et al., "Human ovarian carcinomaassociated mesenchymal stem cells regulate cancer stem cells and tumorigenesis via altered BMP production," Journal of Clinical Investigation, vol. 121, no. 8, pp. 3206-3219, 2011.

[52] J. P. Medema, "Cancer stem cells: the challenges ahead," Nature Cell Biology, vol. 15, no. 4, pp. 338-344, 2013.

[53] M. G. Kelly, A. B. Alvero, R. Chen et al., "TLR-4 signaling promotes tumor growth and paclitaxel chemoresistance in ovarian cancer," Cancer Research, vol. 66, no. 7, pp. 3859-3868, 2006.

[54] R. Chen, A. B. Alvero, D. A. Silasi et al., "Regulation of IKK $\beta$ by miR-199a affects NF- $\kappa$ B activity in ovarian cancer cells," Oncogene, vol. 27, no. 34, pp. 4712-4723, 2008.

[55] L. Cao, M. Shao, J. Schilder, T. Guise, K. S. Mohammad, and D. Matei, "Tissue transglutaminase links TGF-B, epithelial to mesenchymal transition and a stem cell phenotype in ovarian cancer," Oncogene, vol. 31, no. 20, pp. 2521-2534, 2012.

[56] J. Pasquier and A. Rafii, "Role of the microenvironment in ovarian cancer stem cell maintenance," BioMed Research International, vol. 2013, Article ID 630782, 10 pages, 2013.

[57] D. Liang, Y. Ma, J. Liu et al., "The hypoxic microenvironment upgrades stem-like properties of ovarian cancer cells," $B M C$ Cancer, vol. 12, article 201, 2012.

[58] W. K. Chau, C. K. Ip, A. S. C. Mak, H.-C. Lai, and A. S. T. Wong, "C-Kit mediates chemoresistance and tumor-initiating capacity of ovarian cancer cells through activation of Wnt/ $\beta$-cateninATP-binding cassette G2 signaling," Oncogene, vol. 32, no. 22, pp. 2767-2781, 2013.

[59] G. Yin, A. B. Alvero, V. Craveiro et al., "Constitutive proteasomal degradation of TWIST-1 in epithelial-ovarian cancer stem cells impacts differentiation and metastatic potential," Oncogene, vol. 32, no. 1, pp. 39-49, 2013.

[60] R. Chen, M. C. Nishimura, S. M. Bumbaca et al., "A hierarchy of self-renewing tumor-initiating cell types in glioblastoma," Cancer Cell, vol. 17, no. 4, pp. 362-375, 2010.

[61] P. B. Gupta, C. M. Fillmore, G. Jiang et al., "Stochastic state transitions give rise to phenotypic equilibrium in populations of cancer cells," Cell, vol. 146, pp. 633-644, 2011.

[62] D. K. Chan and W. K. Miskimins, "Metformin and phenethyl isothiocyanate combined treatment in vitro is cytotoxic to ovarian cancer cultures," Journal of Ovarian Research, vol. 5, no. 1, article 19, 2012.

[63] R. Erices, M. L. Bravo, P. Gonzalez et al., "Metformin, at concentrations corresponding to the treatment of diabetes, potentiates the cytotoxic effects of carboplatin in cultures of ovarian cancer cells," Reproductive Sciences, vol. 20, pp. 1433-1446, 2013.

[64] R. Rattan, R. P. Graham, J. L. Maguire, S. Giri, and V. Shridhar, "Metformin suppresses ovarian cancer growth and metastasis with enhancement of cisplatin cytotoxicity in Vivo," Neoplasia, vol. 13, no. 5, pp. 483-491, 2011.

[65] A. Yasmeen, M.-C. Beauchamp, E. Piura, E. Segal, M. Pollak, and W. H. Gotlieb, "Induction of apoptosis by metformin in epithelial ovarian cancer: involvement of the Bcl-2 family proteins," Gynecologic Oncology, vol. 121, no. 3, pp. 492-498, 2011. 
[66] R. Rattan, S. Giri, L. C. Hartmann, and V. Shridhar, "Metformin attenuates ovarian cancer cell growth in an AMP-kinase dispensable manner," Journal of Cellular and Molecular Medicine, vol. 15, no. 1, pp. 166-178, 2011.

[67] W. H. Gotlieb, J. Saumet, M.-C. Beauchamp et al., "In vitro metformin anti-neoplastic activity in epithelial ovarian cancer," Gynecologic Oncology, vol. 110, no. 2, pp. 246-250, 2008.

[68] D. Iliopoulos, H. A. Hirsch, and K. Struhl, "Metformin decreases the dose of chemotherapy for prolonging tumor remission in mouse xenografts involving multiple cancer cell types," Cancer Research, vol. 71, no. 9, pp. 3196-3201, 2011.

[69] A. Prigione, B. Lichtner, H. Kuhl et al., "Human induced pluripotent stem cells harbor homoplasmic and heteroplasmic mitochondrial DNA mutations while maintaining human embryonic stem cell-like metabolic reprogramming," Stem Cells, vol. 29, no. 9, pp. 1338-1348, 2011.

[70] A. Vazquez-Martin, L. Vellon, P. M. Quirós et al., "Activation of AMP-activated protein kinase (AMPK) provides a metabolic barrier to reprogramming somatic cells into stem cells," Cell Cycle, vol. 11, no. 5, pp. 974-989, 2012.

[71] A.-C. Buckendahl, J. Budczies, O. Fiehn et al., "Prognostic impact of AMP-activated protein kinase expression in ovarian carcinoma: correlation of protein expression and GC/TOF-MSbased metabolomics," Oncology Reports, vol. 25, no. 4, pp. 1005$1012,2011$.

[72] J.-Y. Lee, I. Jeon, J. W. Kim, Y.-S. Song, J.-M. Yoon, and S. M. Park, "Diabetes mellitus and ovarian cancer risk: a systematic review and meta-analysis of observational studies," International Journal of Gynecological Cancer, vol. 23, no. 3, pp. 402-412, 2013.

[73] A. Bakhru, R. J. Buckanovich, and J. J. Griggs, "The impact of diabetes on survival in women with ovarian cancer," Gynecologic Oncology, vol. 121, no. 1, pp. 106-111, 2011.

[74] M. Jalving, J. A. Gietema, J. D. Lefrandt et al., "Metformin: taking away the candy for cancer?" European Journal of Cancer, vol. 46, no. 13, pp. 2369-2380, 2010.

[75] J. M. M. Evans, L. A. Donnelly, A. M. Emslie-Smith, D. R. Alessi, and A. D. Morris, "Metformin and reduced risk of cancer in diabetic patients," The British Medical Journal, vol. 330, no. 7503, pp. 1304-1305, 2005.

[76] S. Kumar, A. Meuter, P. Thapa et al., "Metformin intake is associated with better survival in ovarian cancer: a case-control study," Cancer, vol. 119, no. 3, pp. 555-562, 2013.

[77] P. Dilokthornsakul, N. Chaiyakunapruk, W. Termrungruanglert, C. Pratoomsoot, S. Saokeaw, and R. Sruamsiri, "The effects of metformin on ovarian cancer: a systematic review," International Journal of Gynecological Cancer, vol. 23, pp. 15441551, 2013.

[78] M. Pollak, "Insulin and insulin-like growth factor signalling in neoplasia," Nature Reviews Cancer, vol. 8, no. 12, pp. 915-928, 2008.

[79] P. Goodwin, K. Pritchard, M. Ennis, M. Clemons, M. Graham, and I. G. Fantus, "Insulin-lowering effects of metformin in women with early breast cancer," Clinical Breast Cancer, vol. 8, no. 6, pp. 501-505, 2008.

[80] M. R. Owen, E. Doran, and A. P. Halestrap, "Evidence that metformin exerts its anti-diabetic effects through inhibition of complex 1 of the mitochondrial respiratory chain," Biochemical Journal, vol. 348, no. 3, pp. 607-614, 2000.

[81] M. N. Pollak, "Investigating metformin for cancer prevention and treatment: the end of the beginning," Cancer Discovery, vol. 2, no. 9, pp. 778-790, 2012.
[82] J. H. No, Y.-T. Jeon, I.-A. Park et al., "Activation of mTOR signaling pathway associated with adverse prognostic factors of epithelial ovarian cancer," Gynecologic Oncology, vol. 121, no. 1, pp. 8-12, 2011.

[83] B. Viollet, B. Guigas, N. Sanz Garcia, J. Leclerc, M. Foretz, and F. Andreelli, "Cellular and molecular mechanisms of metformin: an overview," Clinical Science, vol. 122, no. 6, pp. 253-270, 2012.

[84] R. G. Jones, D. R. Plas, S. Kubek et al., "AMP-activated protein kinase induces a p53-dependent metabolic checkpoint," Molecular Cell, vol. 18, no. 3, pp. 283-293, 2005.

[85] M. Buzzai, R. G. Jones, R. K. Amaravadi et al., "Systemic treatment with the antidiabetic drug metformin selectively impairs p53-deficient tumor cell growth," Cancer Research, vol. 67, no. 14, pp. 6745-6752, 2007.

[86] C. Algire, L. Amrein, M. Bazile, S. David, M. Zakikhani, and M. Pollak, "Diet and tumor LKB1 expression interact to determine sensitivity to anti-neoplastic effects of metformin in vivo," Oncogene, vol. 30, no. 10, pp. 1174-1182, 2011.

[87] I. B. Sahra, K. Laurent, S. Giuliano et al., "Targeting cancer cell metabolism: the combination of metformin and 2-deoxyglucose induces p53-dependent apoptosis in prostate cancer cells," Cancer Research, vol. 70, no. 6, pp. 2465-2475, 2010.

[88] J.-H. Cheong, E. S. Park, J. Liang et al., "Dual inhibition of tumor energy pathway by 2-deoxyglucose and metformin is effective against a broad spectrum of preclinical cancer models," Molecular Cancer Therapeutics, vol. 10, no. 12, pp. 2350-2362, 2011.

[89] A. Priebe, L. Tan, H. Wahl et al., "Glucose deprivation activates AMPK and induces cell death through modulation of Akt in ovarian cancer cells," Gynecologic Oncology, vol. 122, no. 2, pp. 389-395, 2011.

[90] P. S. Tanwar, G. Mohapatra, S. Chiang et al., "Loss of LKB1 and PTEN tumor suppressor genes in the ovarian surface epithelium induces papillary serous ovarian cancer," Carcinogenesis, vol. 35, no. 3, pp. 546-553, 2013.

[91] A. Macciò and C. Madeddu, "Inflammation and ovarian cancer," Cytokine, vol. 58, no. 2, pp. 133-147, 2012.

[92] C. Ersoy, S. Kiyici, F. Budak et al., "The effect of metformin treatment on VEGF and PAI-1 levels in obese type 2 diabetic patients," Diabetes Research and Clinical Practice, vol. 81, no. 1, pp. 56-60, 2008.

[93] S. S. Lund, L. Tarnow, C. D. A. Stehouwer et al., "Impact of metformin versus repaglinide on non-glycaemic cardiovascular risk markers related to inflammation and endothelial dysfunction in non-obese patients with type 2 diabetes," European Journal of Endocrinology, vol. 158, no. 5, pp. 631-641, 2008.

[94] N.-L. Huang, S.-H. Chiang, C.-H. Hsueh, Y.-J. Liang, Y.-J. Chen, and L.-P. Lai, "Metformin inhibits TNF- $\alpha$-induced I $\kappa$ B kinase phosphorylation, I $\kappa$ B- $\alpha$ degradation and IL- 6 production in endothelial cells through PI3K-dependent AMPK phosphorylation," International Journal of Cardiology, vol. 134, no. 2, pp. 169-175, 2009.

[95] A. Salminen, J. M. T. Hyttinen, and K. Kaarniranta, "AMPactivated protein kinase inhibits NF- $\kappa \mathrm{B}$ signaling and inflammation: impact on healthspan and lifespan," Journal of Molecular Medicine, vol. 89, no. 7, pp. 667-676, 2011.

[96] B. Wu, S. Li, L. Sheng et al., "Metformin inhibits the development and metastasis of ovarian cancer," Oncology Reports, vol. 28, no. 3, pp. 903-908, 2012.

[97] H. Liao, Q. Zhou, Y. Gu, T. Duan, and Y. Feng, "Luteinizing hormone facilitates angiogenesis in ovarian epithelial tumor 
cells and metformin inhibits the effect through the mTOR signaling pathway," Oncology Reports, vol. 27, no. 6, pp. 18731878, 2012.

[98] V. Stambolic, J. R. Woodgett, I. G. Fantus, K. I. Pritchard, and P. J. Goodwin, "Utility of metformin in breast cancer treatment, is neoangiogenesis a risk factor?" Breast Cancer Research and Treatment, vol. 114, no. 2, pp. 387-389, 2009.

[99] E. Lonardo, M. Cioffi, P. Sancho et al., "Metformin targets the metabolic achilles heel of human pancreatic cancer stem cells," PLoS ONE, vol. 8, Article ID e76518, 2013. 


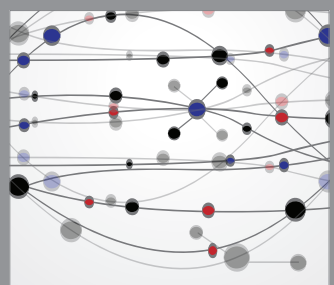

The Scientific World Journal
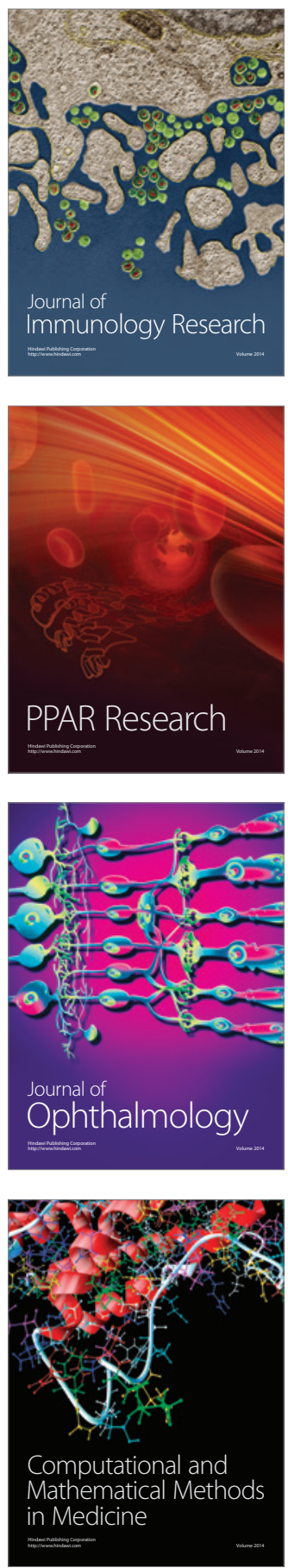

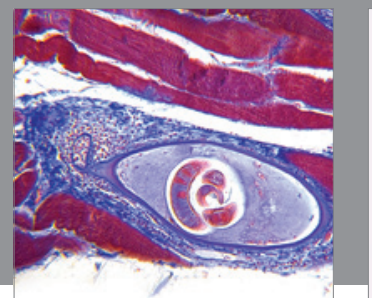

Gastroenterology

Research and Practice
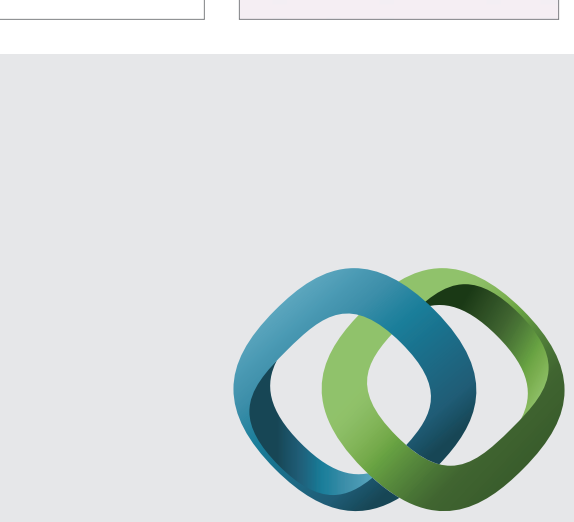

\section{Hindawi}

Submit your manuscripts at

http://www.hindawi.com
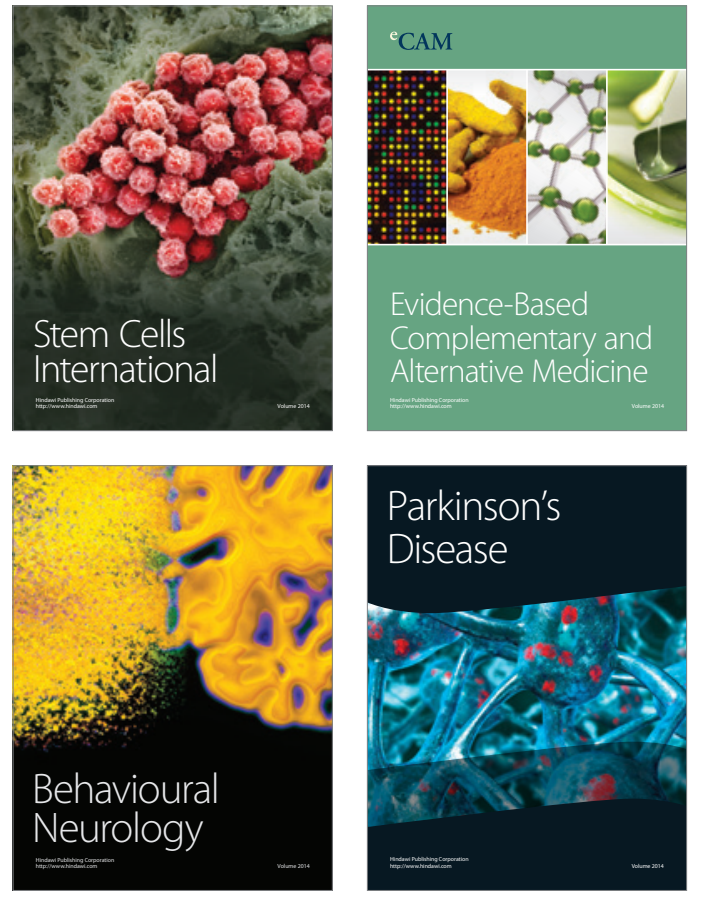
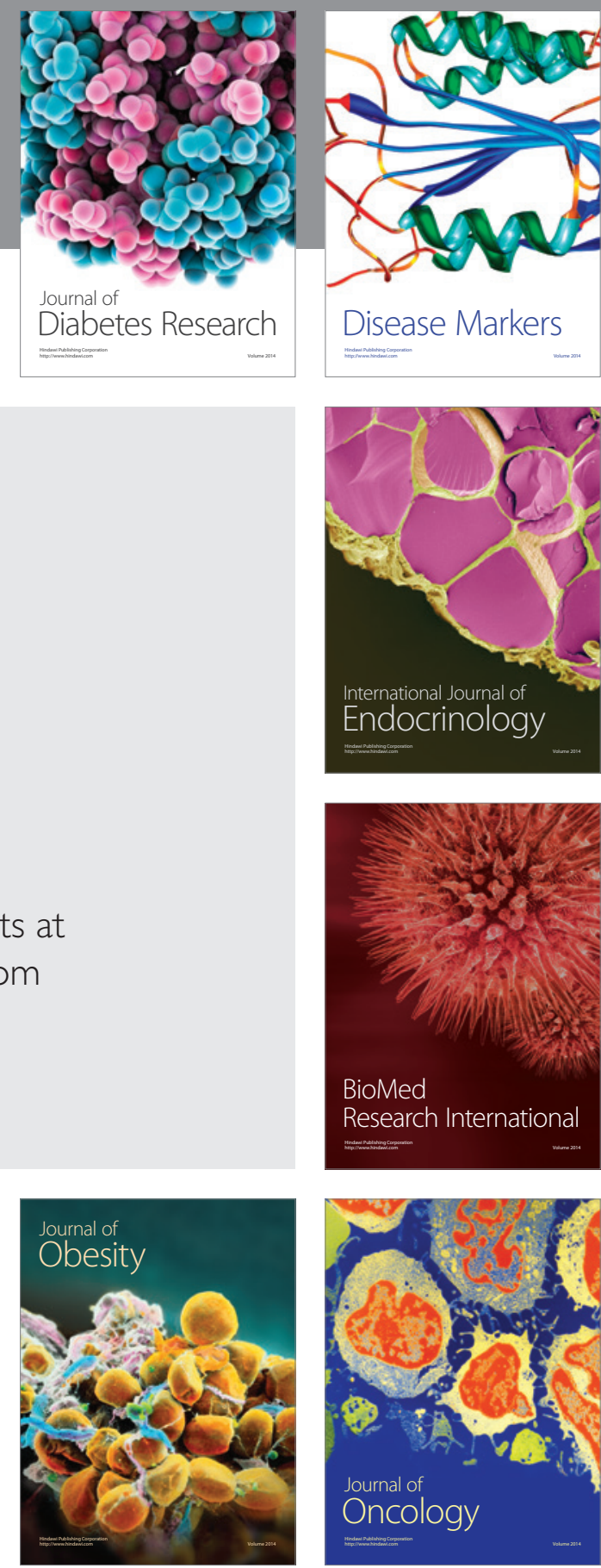

Disease Markers
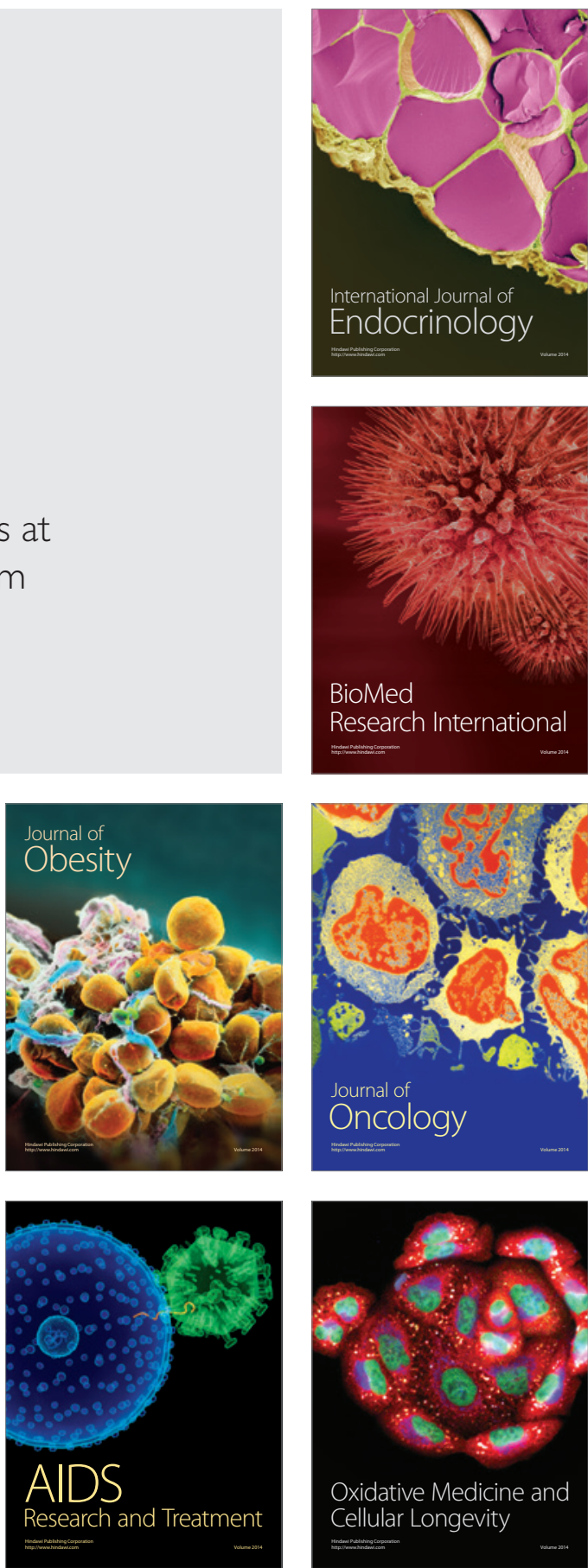